Review Article

\title{
Lights and Shadows about the Effectiveness of IVF in HIV Infected Women: A Systematic Review
}

\author{
Catarina Marques, ${ }^{1}$ Cristina Guerreiro, ${ }^{2}$ and Sérgio Reis Soares ${ }^{3}$ \\ ${ }^{1}$ Maternidade Dr. Alfredo da Costa, Rua Viriato, 1069-089 Lisboa, Portugal \\ ${ }^{2}$ Fetal Maternal Department, Maternidade Dr. Alfredo da Costa, Lisbon, Portugal \\ ${ }^{3}$ Instituto Valenciano de Infertilidade (IVI-Lisboa), Lisbon, Portugal \\ Correspondence should be addressed to Catarina Marques; catarinaomarques@gmail.com
}

Received 14 July 2015; Revised 6 November 2015; Accepted 12 November 2015

Academic Editor: Susan Cu-Uvin

Copyright (C) 2015 Catarina Marques et al. This is an open access article distributed under the Creative Commons Attribution License, which permits unrestricted use, distribution, and reproduction in any medium, provided the original work is properly cited.

\begin{abstract}
Background. HIV infected women have higher rates of infertility. Objective. The purpose of this literature review is to evaluate the effectiveness of fresh IVF/ICSI cycles in HIV infected women. Materials and Methods. A search of the PubMed database was performed to identify studies assessing fresh nondonor oocyte IVF/ICSI cycle outcomes of serodiscordant couples with an HIV infected female partner. Results and Discussion. Ten studies met the inclusion criteria. Whenever a comparison with a control group was available, with the exception of one case, ovarian stimulation cancelation rate was higher and pregnancy rate (PR) was lower in HIV infected women. However, statistically significant differences in both rates were only seen in one and two studies, respectively. A number of noncontrolled sources of bias for IVF outcome were identified. This fact, added to the small size of samples studied and heterogeneity in study design and methodology, still hampers the performance of a meta-analysis on the issue. Conclusion. Prospective matched case-control studies are necessary for the understanding of the specific effects of HIV infection on ovarian response and ART outcome.
\end{abstract}

\section{Introduction}

The human immunodeficiency virus (HIV) epidemic arose from zoonotic infections with simian immunodeficiency viruses from African primates. Since then, the global epidemiology of HIV infection has changed markedly: the prevalence of HIV has increased from 31 million in 2002 to 36.9 million in 2014, essentially due to prolonged survival caused by antiretroviral therapy, whereas the global incidence has decreased from 3.3 million in 2002 to 2 million in 2014 [1].

Since the introduction of antiretroviral therapies, two major medical achievements have been made, allowing many couples with an HIV positive partner to consider pregnancy planning:

(1) Life expectancy of infected patients as well as their life quality has dramatically improved during the last 10 years [2].

(2) A significant reduction in mother-to-child HIV transmission (MTCT) has been observed, especially in developed countries, with transmission rates lower than $1 \%$ to $2 \%$, compared to $14 \%$ to $42 \%$ without any intervention. This has been achieved with the use of antiretroviral drug combinations during pregnancy and labor/delivery, neonatal prophylaxis, elective caesarean delivery, and avoidance of breast feeding [3].

Over $80 \%$ of people infected with HIV are of reproductive age (15 to 44 years old). Reports suggest that there are currently more than 140,000 HIV serodiscordant heterosexual couples in the United States (US), approximately 50\% of whom having reproductive plans [4]. According to the National Perinatal HIV Hotline and Clinicians Network, calls pertaining to HIV serodiscordant couples and their options for safer conception have increased significantly between 2006 and 2011 [5].

Managing HIV infected patients with a childbearing wish involves a multidisciplinary approach, ideally including maternal-fetal medicine specialists, HIV/AIDS specialists, neonatologists, pediatricians, psychiatrists, social workers, 
and reproductive endocrinologists [6]. Preconception counseling is highly recommended among HIV serodiscordant and seroconcordant couples, allowing them to make more informed choices in order to reduce sexual transmission and improving pregnancy outcome [7]. However, a recent survey of HIV infected women who had been or were pregnant at the time of the questionnaire showed that more than half of them did not have preconception counseling [8]. Evaluating the need for antiretroviral therapy should be part of the initial assessment of preconception counseling. Any concurrent sexually transmitted infection should be treated and safe sexual practices should be encouraged [9].

Infertility affects approximately $15 \%$ of the general population and HIV infected patients, both men and women, have higher rates of infertility than their HIV negative counterparts $[10,11]$.

In serodiscordant couples in which the male partner is infected, assisted reproductive technology (ART) is the safest way to prevent sexual transmission. After the spermwashing (SW) procedure, there are two main options to achieve a pregnancy: intrauterine insemination (IUI) and in vitro fertilization (IVF)/intracytoplasmic sperm injection (ICSI). In couples with a normal fertility evaluation, IUI is an effective approach. If semen analysis is abnormal, then IVF/ICSI is undoubtedly the treatment to be offered [6]. SW eliminates round cells, seminal plasma, and the majority of immotile sperm. Spermatozoa are isolated by sequential density gradient and swim-up techniques and are subsequently tested by PCR assays for the presence of HIV RNA [12]. A systematic review and meta-analysis summarized the experience with serodiscordant couples with an infected male partner until 2013, with 2,393 SW-IUI and 780 SW-IVF treatment cycles documented [13]. The authors concluded that HIV infected men with noninfected partners have pregnancy and live birth rates with ART comparable to seronegative couples.

In serodiscordant couples in which the female partner is infected, pregnancy can be achieved without the risk of sexual transmission by self-insemination around the time of ovulation [6]. If conception does not occur after more than six cycles of self-insemination, or if a preexisting fertility problem was diagnosed, the use of ART should be envisaged [7].

Most of the reports so far published on IUI or IVF treatments performed in serodiscordant couples refer to infected male partners. Very few studies have addressed IVF outcome in serodiscordant couples with an HIV infected female partner. The purpose of this review is to evaluate the effectiveness of fresh nondonor oocyte IVF/ICSI treatments performed in this population. Control over variables that are traditionally known to influence IVF/ICSI outcome, such as female age, ovarian reserve, race/ethnicity, Body Mass Index (BMI), tobacco consumption, the presence of tubal disease, and the number of embryos replaced in the uterus, was ascertained in the studies found.

\section{Materials and Methods}

A search of the PubMed database was performed in order to identify all studies involving ART including the HIV infected population published until July 2014. The search terms used were "HIV" AND "assisted reproduction," "HIV" AND "assisted reproductive technology," "HIV" AND "in vitro fertilization," and "HIV" AND "infertility". Abbreviations such as "IVF" and "ICSI" were also used. An initial list of 626 studies was obtained. Inclusion criterion was studies assessing fresh nondonor oocyte IVF/ICSI cycle outcomes of serodiscordant couples with an HIV infected female partner. References with abstracts that demonstrated them to be unrelated to the IVF/ICSI cycle outcomes of serodiscordant couples with an HIV infected female partner were excluded without full text assessment, as were reviews and case reports. All original articles with abstracts that indicated them to be within the scope of this study were fully assessed; when this assessment was confirmed, they were included in the review. Articles in languages other than English, Portuguese, Spanish, or French were excluded. Ten studies were finally included. Figure 1 summarizes the steps involved in literature selection based on Preferred Reporting Items for Systematic Reviews and Meta-Analyses (PRISMA) guidelines [24].

\section{Results}

Studies analyzed reported on ART treatments performed in a total of $342 \mathrm{HIV}$ infected women, with a mean age of 35.4 years, who underwent 516 IVF/ICSI cycles (Table 1). The average CD4 count ranged from " $>200$ ” to 712 cells $/ \mathrm{mm}^{3}$, $48 \%$ to $100 \%$ of patients in each study had undetectable viral loads, and $44 \%$ to $95 \%$ of them were being treated with combined antiretroviral therapy.

Table 1 shows baseline characteristics of study and control groups and Table 2 shows the outcomes of ovarian stimulation and IVF.

Among the studies included in this review, data concerning ovarian response to stimulation in HIV infected patients can be summarized as follows: some of the initial studies report the need of higher doses of gonadotropin to achieve satisfactory ovarian response (Terriou et al. 2005 [16]; Coll et al. 2006 [17]), while a normal response to stimulation is described in infected women who are in good general health conditions and reach egg pick-up (Martinet et al. 2006 [18]). Data from most recent studies suggest that a normal ovarian response is seen in these patients (Manigart et al. 2006 [19], Douglas et al. 2009 [20], Prisant et al. 2010 [21], Santulli et al. 2011 [22], and Nurudeen et al. 2013 [23]) (Table 2). However, in the nine studies that assessed the cancellation rate of ovarian stimulation in HIV infected patients, although significance was observed in only one of them (15.2\% versus $4.9 \%$ in the control group), in all the instances in which a comparison could be made cancellation rate was higher in the study group than in controls (Table 2).

In study groups, the clinical PR per stimulation cycle initiated varied from $6.7 \%$ to $24.1 \%$ and the clinical PR per embryo transfer varied from $9.1 \%$ to $63 \%$ (Table 2). Unfortunately, not all studies mentioned the rate per cycle initiated. A summary of the conclusions of the six studies that compared the PRs in HIV infected women with those from noninfected controls is (Table 2) as follows: 


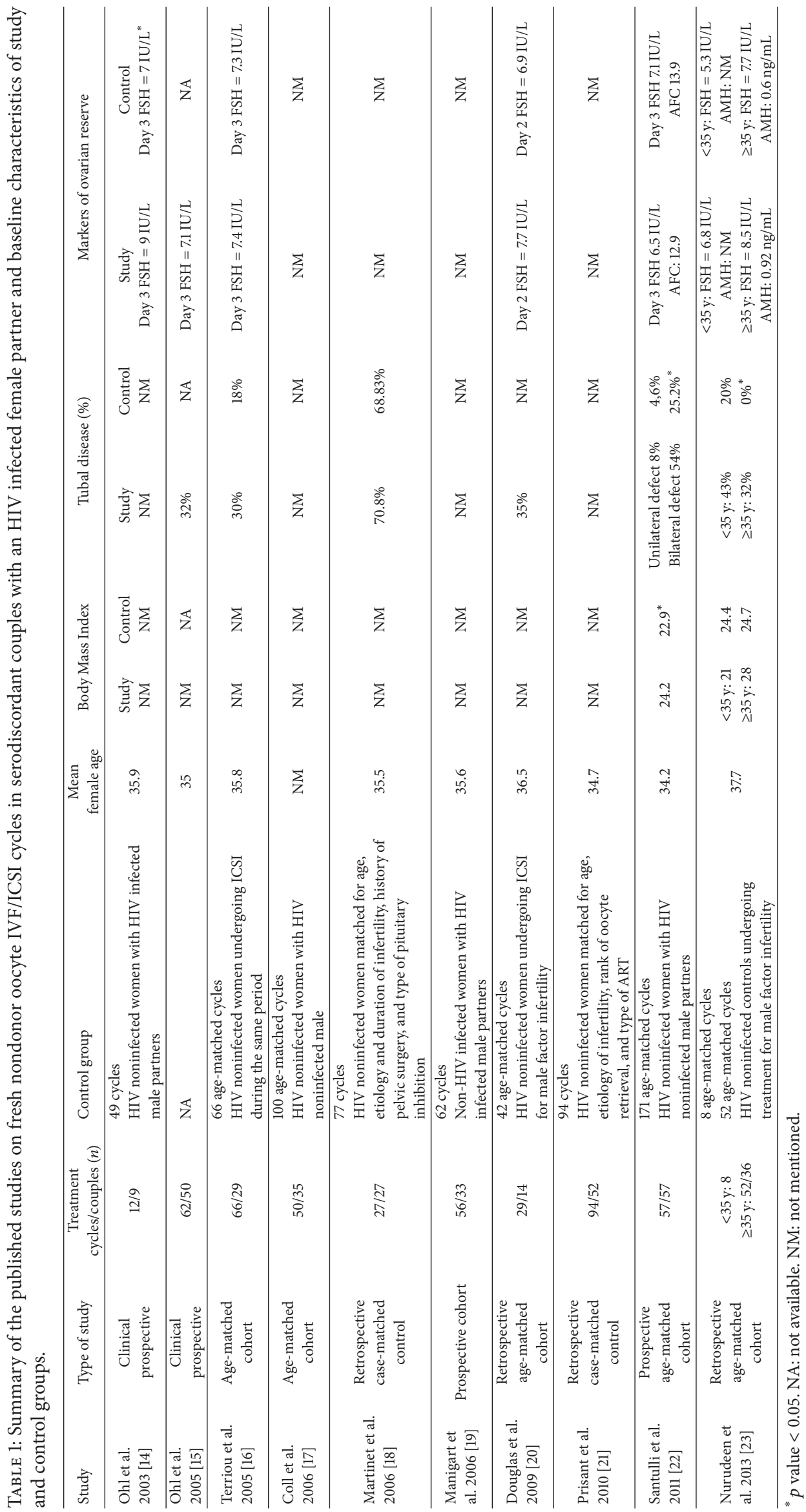




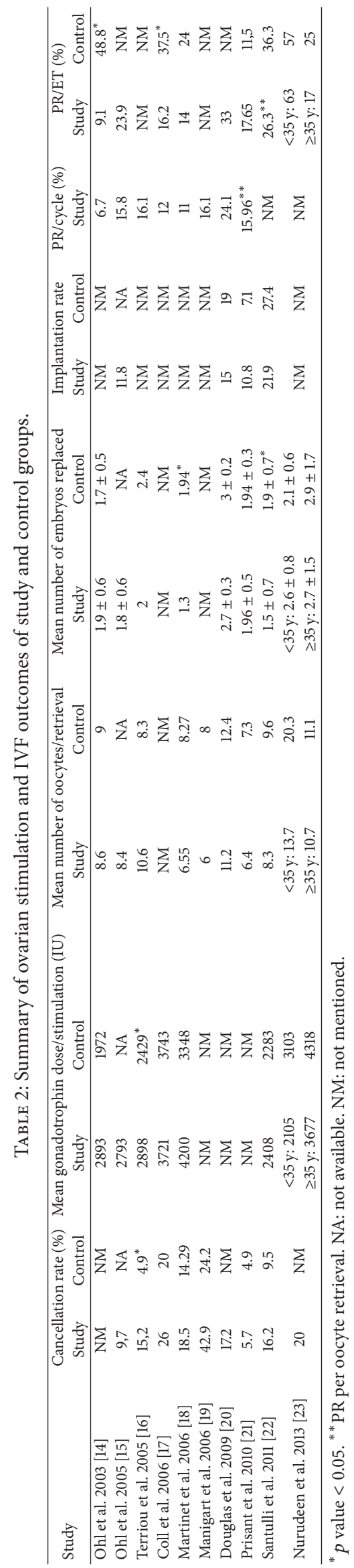




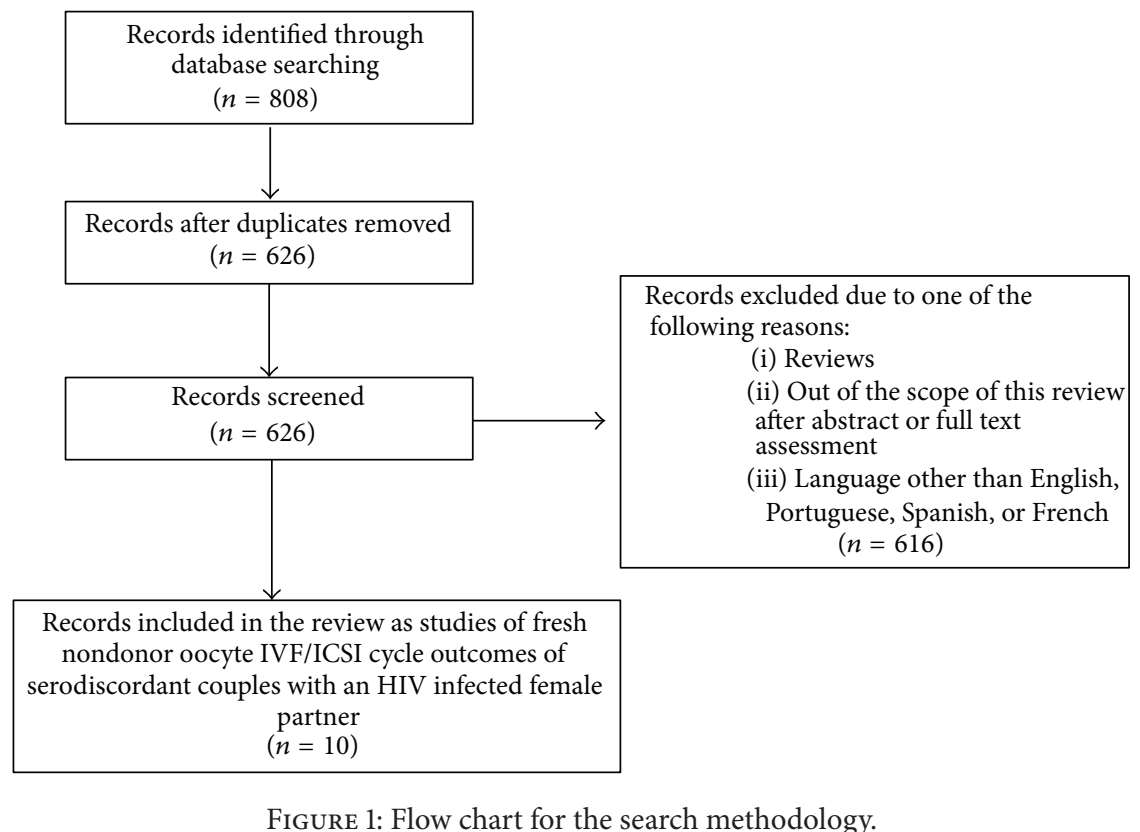

(i) In two studies, HIV infected women had a statistically significantly lower PR (Ohl et al. 2003 [14], Coll et al. 2006 [17]).

(ii) In three studies, the PR of HIV infected women was not statistically significantly different from that of control subjects, but lower values were observed (Martinet et al. 2006 [18], Santulli 2011 et al. [22], and Nurudeen et al. 2013 [23]).

(iii) In one study, the PR of HIV infected women was not statistically significantly different from that of control subjects, but a higher value was observed (Prisant et al. 2010 [21]).

In all studies, PR was reported per embryo transfer, with the exception of two cases of PR per cycle initiated $[16,19]$ and one of PRs per oocyte retrieval [22]. Noteworthy, vertical transmission of HIV infection was zero.

Concerning variables that are traditionally known to influence IVF/ICSI outcome, data from studies can be summarized as follows.

3.1. Female Age. Seven studies included in this review were age-matched. In only one study, ART results were stratified by age: in both age groups ( $<35$ and $\geq 35$ years), infected and control patients had similar IVF/ICSI clinical outcomes with similar clinical PRs per embryo transfer [23].

3.2. Ovarian Reserve. Six studies included in this review evaluated the ovarian reserve, but only five of them had a control group (Table 1):

(i) In one of them, the comparison between early follicular phase serum FSH of HIV infected women and controls showed a statistically significant difference $(9.0 \pm 2.4$ versus $7.0 \pm 2.9 \mathrm{IU} / \mathrm{L}$, resp., $p<0.001)(\mathrm{Ohl}$ et al. 2003 [14]). (ii) In the other four studies, HIV infected women and controls had similar values for markers of ovarian reserve. FSH and HAM levels and antral follicle count (AFC) were evaluated (Terriou et al. 2005 [16]; Douglas et al. 2005 [20]; Santulli et al. 2011 [22]; Nurudeen et al. 2013 [23]).

3.3. Race/Ethnicity. In our review, only two studies documented patients' race/ethnicity $[18,23]$. In both of them, the proportion of black women was significantly higher in the study group and this was mentioned as a possible source of bias.

3.4. Body Mass Index (BMI). Only two of the studies analyzed evaluated BMI (Table 1):

(i) In one of them, HIV infected women had higher BMI than control subjects (24.2 versus $22.9, p=0.032$ ) (Santulli et al. 2011 [22]).

(ii) In the other one, HIV infected women and controls had similar BMI (Nurudeen et al. 2013 [23]).

3.5. Tobacco. Of the studies analyzed in this review, only two controlled for tobacco consumption:

(i) In the first of them, no differences were found between HIV infected women and controls (Santulli et al. 2011 [22]).

(ii) In the other one, in the group of women $\geq 35$ years of age, infected patients smoked significantly more often than controls $(16 \%$ versus $0 \% ; p<0.05)$. Although parameters of ovarian reserve, the number of mature oocytes retrieved, and fertilization and clinical PRs per embryo transfer were similar in HIV infected women and controls, live birth rates per embryo 
transfer were significantly lower for HIV infected women (6\% versus $24 \%, p=0.04$ ) (Nurudeen et al. 2013 [23]). Unfortunately, cancelation rates were not compared between groups.

3.6. Tubal Disease. In four of the studies reviewed, the incidence of tubal disease could be compared between the study and control groups and in all of them a higher proportion of tubal disease in HIV infected women was documented, confirming previous reports on the literature [25] (Table 1). In two of them, a statistically significant difference was observed (Santulli et al. 2011 [22] and Nurudeen et al. 2013 [23]).

3.7. Number of Embryos Replaced per Transfer. In the studies analyzed, different criteria were adopted regarding embryo transfer (Table 2). In three of them, the authors reported they were more likely to replace a lower number of embryos in infected women (Terriou et al. 2005 [16]; Martinet et al. 2006 [18]; Santulli et al. 2011 [22]). However, documentation of a significant reduction in the mean number of embryos transferred was seen in only two of these (Martinet et al. 2006 [18]; Santulli et al. 2011 [22]).

\section{Discussion}

The variability of results observed in the studies analyzed can be mostly related to the small size of the samples studied, heterogeneity in study design, and methodology and incomplete control over potential confounding data. These limitations do not allow for the implementation of a statistical approach that might lead to solid conclusions, such as a metaanalysis.

Variables that are traditionally known to influence IVF/ICSI outcome and should therefore be controlled for are the following:

(1) Female age.

(2) Ovarian reserve.

(3) Race/ethnicity.

(4) Body Mass Index (BMI).

(5) Tobacco consumption.

(6) Tubal disease.

(7) Number of embryos replaced.

A summary of the variables controlled per study is available in Table 3 and aspects to be considered regarding each of them are as follows.

4.1. Female Age. Population studies from areas where no consistent methods of birth control are applied show that natural fertility starts to decline after the age of 30 , has its decline accelerated in the mid-30s, and ends at a mean age of 41 years [26]. The age-related effect on female fertility has also been shown in numerous reports on the results of IVF treatments due to a progressive decline on oocyte quality and quantity. The implantation rate per embryo clearly decreases after the age of 35 and the same has been shown for the probability of a live birth in IVF [27].
Due to this knowledge, control over female age is of paramount importance in any study on IVF treatment outcome. Such was not seen in three of the ten studies included in this review.

4.2. Ovarian Reserve. ART requires controlled ovarian stimulation for the achievement of improved efficacy. Currently, it is not consensual that HIV infection affects ovarian reserve. Seifer et al. evaluated the markers of ovarian follicular reserve and reproductive ageing in $187 \mathrm{HIV}$ infected women not diagnosed as infertile [28]. Early follicular phase FSH, estradiol, inhibin $\mathrm{B}$, and anti-Mullerian hormone (AMH) levels were measured. No evidence was found that HIV infection affects ovarian ageing. On the other hand, Ohl et al. measured serum FSH, inhibin $\mathrm{B}, \mathrm{AMH}$, and the antral follicle count (AFC) in 78 HIV infected women [29]. Mean FSH was $36 \%$ higher than that seen in the control group, whereas mean inhibin B and $\mathrm{AMH}$ were $57 \%$ and $23 \%$ lower, respectively. AFC was also significantly lower in the study group.

The possible influence of combined antiretroviral therapy on ovarian reserve and ovarian response to stimulation is not clear either. Side effects of the use of antiretroviral drugs such as mitochondrial dysfunction or modification in the lipid metabolism and insulin resistance could have consequences on folliculogenesis and ovulation regulatory processes $[2,30]$. Oocytes from infertile HIV infected women on combined antiretroviral therapy were reported to have $32 \%$ depletion in mtDNA in comparison to infertile uninfected controls $(p<$ $0.05)$ and depletion was even stronger in patients who failed to become pregnant in IVF treatments [31].

4.3. Race/Ethnicity. A growing number of studies have investigated the association between race/ethnicity and ART outcomes. Most of the studies have focused on comparisons between white Caucasian and black women and some have identified that the last group is more likely to have a diagnosis of tubal factor infertility, leiomyoma/uterine factor infertility, a longer duration of infertility before ART, and higher miscarriage/stillbirth rates [32-36]. Race/ethnicity as a risk factor for poor ART outcomes has been consistently acknowledged in recent large studies, even after adjusting for many confounding factors [37]. This may certainly be a source of bias for data collected in this review.

4.4. Body Mass Index (BMI). Overweight and obesity are well-described risk factors for infertility, particularly as they relate to ovulation disorders. In spite of conflicting results of studies regarding the effect of high BMI on ART outcome, a systematic review and meta-analysis published in 2011 showed that overweight or obese women (BMI $\geq 25)$ had significantly lower clinical pregnancy $(\mathrm{RR}=0.90,95 \% \mathrm{CI}$ $0.85-0.94)$ and live birth rates ( $R R=0.84,95 \%$ CI $0.77-0.92)$ and a significantly higher miscarriage rate $(\mathrm{RR}=1.31,95 \%$ CI 1.18-1.45) [38]. The analysis of overweight women alone $(\mathrm{BMI} \geq 25-29.9)$ also showed lower clinical pregnancy $(\mathrm{RR}=$ $0.91,95 \%$ CI $0.86-0.96)$ and live birth rates $(\mathrm{RR}=0.91,95 \%$ CI $0.85-0.98)$ and higher miscarriage rate $(\mathrm{RR}=1.24,95 \% \mathrm{CI}$ $1.13-1.35)$, compared to women with $\mathrm{BMI}<25$. In conclusion, increased $\mathrm{BMI}$ is associated with adverse outcomes in women 


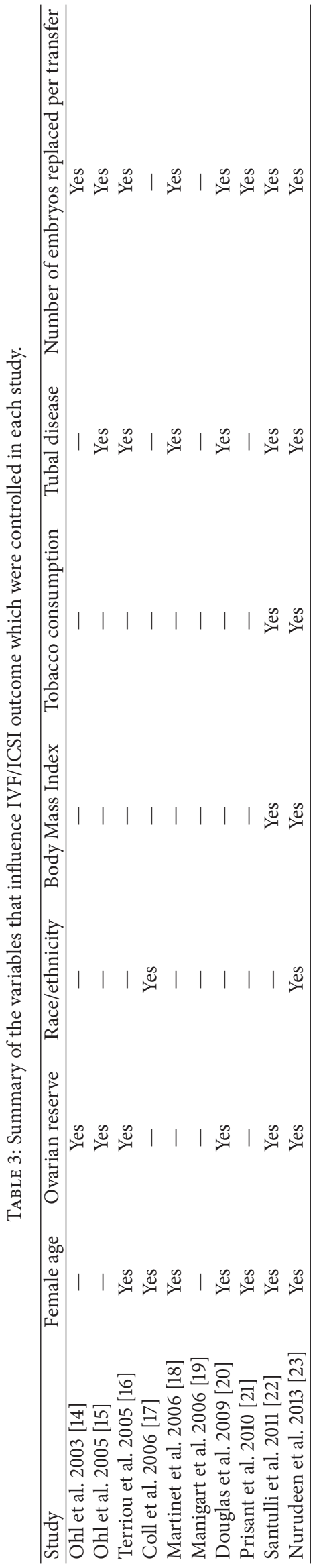


undergoing IVF/ICSI treatment, including lower live birth rates.

Increasing overweight and obesity in the HIV infected population were not seen until two decades ago. This relatively new reality has been attributed to several factors, such as better health due to combined antiretroviral therapy and improved life expectancy, which leads to obesity trends similar to those seen in the general population [39]. This parameter was not properly controlled for in the majority of studies analyzed here.

4.5. Tobacco. Over the last few decades, the prevalence of cigarette smoking among women of reproductive age has increased, reaching, in 2006, 33\% in Europe and 28\% in the USA [40], and is highly prevalent among persons infected with HIV [41]. Studies on IVF showed that cigarette smoking has deleterious effects on many aspects of treatment: ovarian responsiveness to gonadotropins, number of oocytes retrieved, fertilization, implantation, and early placentation [42].

4.6. Tubal Disease. In the studies reviewed, a higher proportion of tubal disease in HIV infected women was documented, confirming previous reports on the literature [25]. It is important to notice that many studies collected data at times during which it was still not a standard procedure to remove hydrosalpinx before IVF, which may have reduced live birth rates and constituted a source of bias [43].

Another factor associated with tubal disease that should be considered is the personal history of pelvic inflammatory disease. This condition has been shown to reduce ovarian response to stimulation due to direct damage to the ovaries and follicle loss or due to mechanical alterations of follicular development [18].

4.7. Number of Embryos Replaced per Transfer. The rate of preterm delivery and multiple gestations after ART is of particular concern for HIV infected women. Preterm labor or premature rupture of membranes may increase the risk of HIV vertical transmission [44]. Elective single embryo transfer for HIV infected women shall be considered in order to reduce the risk of multiple gestations [15]. A lower number of embryos replaced in HIV positive women may obviously contribute to lower pregnancy and live birth rates per transfer.

\section{Conclusions}

Data on PR in IVF/ICSI treatments performed in couples with HIV infected women are conflicting and it is still not clear if these patients display worse clinical outcomes per cycle initiated when compared to the general population. The same can be said specifically about ovarian response to stimulation, though a tendency for higher cancellation rates seems to be observed.

The small size of the samples studied and heterogeneity in study design and methodology make it difficult to draw clear conclusions about the impact of HIV infection in women on IVF outcome. Incomplete control over confounding variables (such as age, race/ethnicity, BMI, tobacco consumption, tubal disease, history of pelvic inflammatory disease, duration of infertility, and the number of embryos replaced) is of special concern. Altogether, such limitations hamper the performance of a proper meta-analysis.

In the future, prospective matched case-control studies are needed to understand the specific effects of HIV infection on ovarian response and ART outcome. Available data suggest some impact of HIV infection on ovarian function and IVF outcome, but noncontrolled sources of bias in published studies do not allow for definitive conclusions.

\section{Conflict of Interests}

The authors declare that there is no conflict of interests regarding the publication of this paper.

\section{References}

[1] UNAIDS, How AIDS Changed Everything, UNAIDS, Geneva, Switzerland, 2015.

[2] Y. Englert, B. Lesage, J.-P. Van Vooren et al., "Medically assisted reproduction in the presence of chronic viral diseases," Human Reproduction Update, vol. 10, no. 2, pp. 149-162, 2004.

[3] A. P. Kourtis and M. Bulterys, "Mother-to-child transmission of HIV: pathogenesis, mechanisms and pathways," Clinics in Perinatology, vol. 37, no. 4, pp. 721-737, 2010.

[4] M. A. Lampe, D. K. Smith, G. J. E. Anderson, A. E. Edwards, and S. R. Nesheim, "Achieving safe conception in HIV-discordant couples: the potential role of oral preexposure prophylaxis (PrEP) in the United States," American Journal of Obstetrics and Gynecology, vol. 204, no. 6, pp. 488.e1-488.e8, 2011.

[5] S. Weber, J. F. Waldura, and D. Cohan, "Safer conception options for HIV serodifferent couples in the United States: the experience of the National Perinatal HIV Hotline and Clinicians' Network," Journal of Acquired Immune Deficiency Syndromes, vol. 63, no. 4, pp. e140-e141, 2013.

[6] S. J. Estes and E. S. Ginsburg, "Use of assisted reproduction in HIV and hepatitis infected couples," UpToDate, 2014.

[7] V. Savasi, L. Mandia, A. Laoreti, and I. Cetin, "Reproductive assistance in HIV serodiscordant couples," Human Reproduction Update, vol. 19, no. 2, pp. 136-150, 2013.

[8] K. E. Squires, S. L. Hodder, J. Feinberg et al., "Health needs of HIV-infected women in the United States: insights from the women living positive survey," AIDS Patient Care and STDs, vol. 25 , no. 5, pp. 279-285, 2011.

[9] V. A. Kushnir and W. Lewis, "Human immunodeficiency virus/acquired immunodeficiency syndrome and infertility: emerging problems in the era of highly active antiretrovirals," Fertility and Sterility, vol. 96, no. 3, pp. 546-553, 2011.

[10] G. F. Homan, M. Davies, and R. Norman, "The impact of lifestyle factors on reproductive performance in the general population and those undergoing infertility treatment: a review," Human Reproduction Update, vol. 13, no. 3, pp. 209223, 2007.

[11] V. A. Moragianni, "Why are we still, 20 years later, depriving human immunodeficiency virus-serodiscordant couples of equal access to fertility care?" Fertility and Sterility, vol. 102, no. 2, pp. 352-353, 2014. 
[12] A. C. Eke and C. Oragwu, "Sperm washing to prevent HIV transmission from HIV-infected men but allowing conception in sero-discordant couples," Cochrane Database of Systematic Reviews, no. 1, Article ID CD008498, 2011.

[13] A. Barnes, D. Riche, L. Mena et al., "Efficacy and safety of intrauterine insemination and assisted reproductive technology in populations serodiscordant for human immunodeficiency virus: a systematic review and meta-analysis," Fertility and Sterility, vol. 102, no. 2, pp. 424-434, 2014.

[14] J. Ohl, M. Partisani, C. Wittemer et al., "Assisted reproduction techniques for HIV serodiscordant couples: 18 months of experience," Human Reproduction, vol. 18, no. 6, pp. 1244-1249, 2003.

[15] J. Ohl, M. Partisani, C. Wittemer, J.-M. Lang, S. Viville, and R. Favre, "Encouraging results despite complexity of multidisciplinary care of HIV-infected women using assisted reproduction techniques," Human Reproduction, vol. 20, no. 11, pp. 31363140, 2005.

[16] P. Terriou, P. Auquier, V. Chabert-Orsini et al., "Outcome of ICSI in HIV-1-infected women," Human Reproduction, vol. 20, no. 10, pp. 2838-2843, 2005.

[17] O. Coll, A. Suy, F. Figueras et al., "Decreased pregnancy rate after in-vitro fertilization in HIV-infected women receiving HAART,' AIDS, vol. 20, no. 1, pp. 121-123, 2006.

[18] V. Martinet, Y. Manigart, S. Rozenberg, B. Becker, M. Gerard, and A. Delvigne, "Ovarian response to stimulation of HIVpositive patients during IVF treatment: a matched, controlled study," Human Reproduction, vol. 21, no. 5, pp. 1212-1217, 2006.

[19] Y. Manigart, S. Rozenberg, P. Barlow, M. Gerard, E. Bertrand, and A. Delvigne, "ART outcome in HIV-infected patients," Human Reproduction, vol. 21, no. 11, pp. 2935-2940, 2006.

[20] N. C. Douglas, J. G. Wang, B. Yu, S. Gaddipati, M. M. Guarnaccia, and M. V. Sauer, "A systematic, multidisciplinary approach to address the reproductive needs of HIV-seropositive women," Reproductive BioMedicine Online, vol. 19, no. 2, pp. 257-263, 2009.

[21] N. Prisant, R. Tubiana, G. Lefebvre et al., "HIV-1 or hepatitis $\mathrm{C}$ chronic infection in serodiscordant infertile couples has no impact on infertility treatment outcome," Fertility and Sterility, vol. 93, no. 3, pp. 1020-1023, 2010.

[22] P. Santulli, V. Gayet, P. Fauque et al., "HIV-positive patients undertaking ART have longer infertility histories than agematched control subjects," Fertility and Sterility, vol. 95, no. 2, pp. 507-512, 2011.

[23] S. K. Nurudeen, L. C. Grossman, L. Bourne, M. M. Guarnaccia, M. V. Sauer, and N. C. Douglas, "Reproductive outcomes of HIV seropositive women treated by assisted reproduction," Journal of Women's Health, vol. 22, no. 3, pp. 243-249, 2013.

[24] D. Moher, A. Liberati, J. Tetzlaff, and D. G. Altman, "Preferred reporting items for systematic reviews and meta-analyses: the PRISMA statement," Annals of Internal Medicine, vol. 151, no. 4, pp. 264-269, 2009.

[25] J. D. Sobel, "Gynecologic infections in human immunodeficiency virus-infected women," Clinical Infectious Diseases, vol. 31, no. 5, pp. 1225-1233, 2000.

[26] E. R. te Velde and P. L. Pearson, "The variability of female reproductive ageing," Human Reproduction Update, vol. 8, no. 2, pp. 141-154, 2002.
[27] F. J. Broekmans, J. Kwee, D. J. Hendriks, B. W. Mol, and C. B. Lambalk, "A systematic review of tests predicting ovarian reserve and IVF outcome," Human Reproduction Update, vol. 12, no. 6, pp. 685-718, 2006.

[28] D. B. Seifer, E. T. Golub, G. Lambert-Messerlian et al., "Biologic markers of ovarian reserve and reproductive aging: application in a cohort study of HIV infection in women," Fertility and Sterility, vol. 88, no. 6, pp. 1645-1652, 2007.

[29] J. Ohl, M. Partisani, C. Demangeat, F. Binder-Foucard, I. Nisand, and J.-M. Lang, "Alterations of ovarian reserve tests in Human Immunodeficiency Virus (HIV)-infected women," Gynecologie Obstetrique Fertilite, vol. 38, no. 5, pp. 313-317, 2010.

[30] C. de Mendoza, M. Sanchez-Conde, E. Ribera, P. Domingo, and V. Soriano, "Could mitochondrial DNA quantitation be a surrogate marker for drug mitochondrial toxicity?" AIDS Reviews, vol. 6, no. 3, pp. 169-180, 2004.

[31] S. López, O. Coll, M. Durban et al., "Mitochondrial DNA depletion in oocytes of HIV-infected antiretroviral-treated infertile women," Antiviral Therapy, vol. 13, no. 6, pp. 833-838, 2008.

[32] F. I. Sharara and H. D. McClamrock, "Differences in in vitro fertilization (IVF) outcome between white and black women in an inner-city, university-based IVF program," Fertility and Sterility, vol. 73, no. 6, pp. 1170-1173, 2000.

[33] E. C. Feinberg, F. W. Larsen, W. H. Catherino, J. Zhang, and A. Y. Armstrong, "Comparison of assisted reproductive technology utilization and outcomes between Caucasian and African American patients in an equal-access-to-care setting," Fertility and Sterility, vol. 85, no. 4, pp. 888-894, 2006.

[34] D. B. Seifer, R. Zackula, D. A. Grainger, and Society for Assisted Reproductive Technology Writing Group Report, "Trends of racial disparities in assisted reproductive technology outcomes in black women compared with white women: Society for Assisted Reproductive Technology 1999 and 2000 vs. 20042006," Fertility and Sterility, vol. 93, no. 2, pp. 626-635, 2010.

[35] D. B. Seifer, L. M. Frazier, and D. A. Grainger, "Disparity in assisted reproductive technologies outcomes in black women compared with white women," Fertility and Sterility, vol. 90, no. 5, pp. 1701-1710, 2008.

[36] V. Y. Fujimoto, B. Luke, M. B. Brown et al., "Racial and ethnic disparities in assisted reproductive technology outcomes in the United States," Fertility and Sterility, vol. 93, no. 2, pp. 382-390, 2010.

[37] S. F. Butts and D. B. Seifer, "Racial and ethnic differences in reproductive potential across the life cycle," Fertility and Sterility, vol. 93, no. 3, pp. 681-690, 2010.

[38] V. Rittenberg, S. Seshadri, S. K. Sunkara, S. Sobaleva, E. OtengNtim, and T. El-Toukhy, "Effect of body mass index on IVF treatment outcome: an updated systematic review and metaanalysis," Reproductive BioMedicine Online, vol. 23, no. 4, pp. 421-439, 2011.

[39] N. Crum-Cianflone, R. Tejidor, S. Medina, I. Barahona, and A. Ganesan, "Obesity among patients with HIV: the latest epidemic," AIDS Patient Care and STDs, vol. 22, no. 12, pp. 925930, 2008.

[40] C. Dechanet, T. Anahory, J. C. Mathieu Daude et al., "Effects of cigarette smoking on reproduction," Human Reproduction Update, vol. 17, no. 1, pp. 76-95, 2011.

[41] N. R. Reynolds, "Cigarette smoking and HIV: more evidence for action," AIDS Education and Prevention, vol. 21, no. 3, pp. 106121, 2009. 
[42] The Practice Committee of the American Society for Reproductive Medicine, "Smoking and infertility: a committee opinion," Fertility and Sterility, vol. 98, no. 6, pp. 1400-1406, 2012.

[43] P. Devroey, B. C. J. M. Fauser, and K. Diedrich, "Approaches to improve the diagnosis and management of infertility," Human Reproduction Update, vol. 15, no. 4, pp. 391-408, 2009.

[44] L. Mandelbrot, M. J. Mayaux, A. Bongain et al., "Obstetric factors and mother-to-child transmission of human immunodeficiency virus type 1: the French perinatal cohorts. SEROGEST French Pediatric HIV Infection Study Group," American Journal of Obstetrics \& Gynecology, vol. 175, no. 3, part 1, pp. 661-667, 1996. 


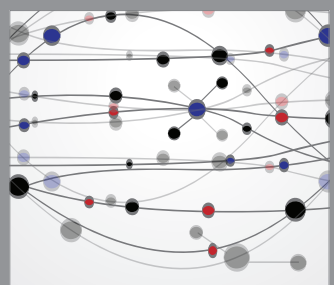

The Scientific World Journal
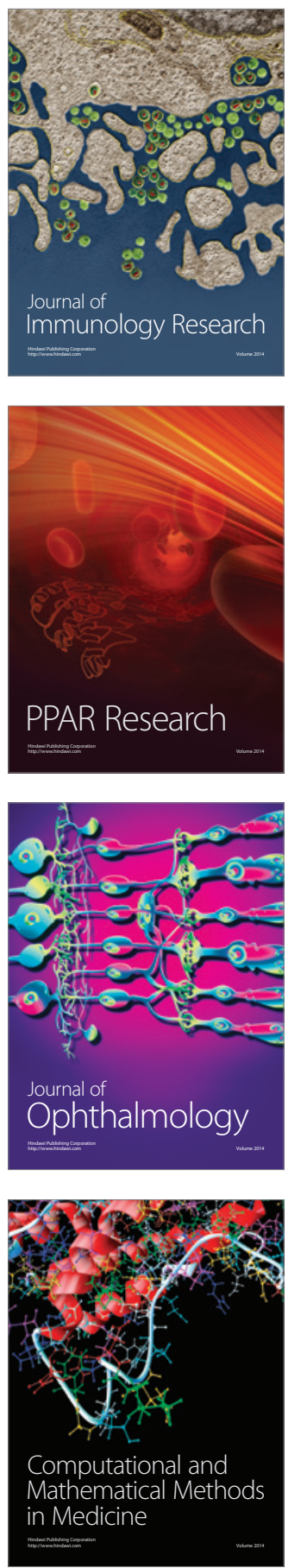

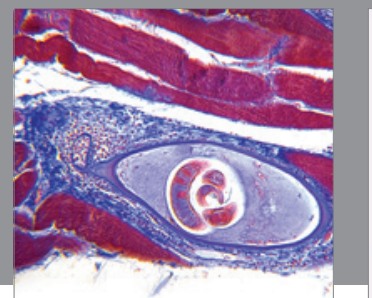

Gastroenterology

Research and Practice
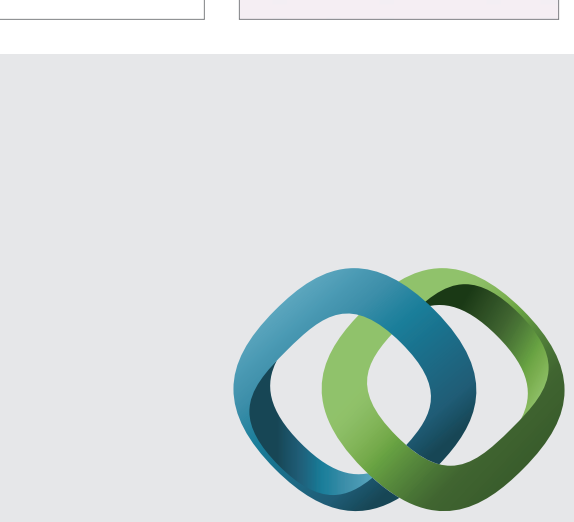

\section{Hindawi}

Submit your manuscripts at

http://www.hindawi.com
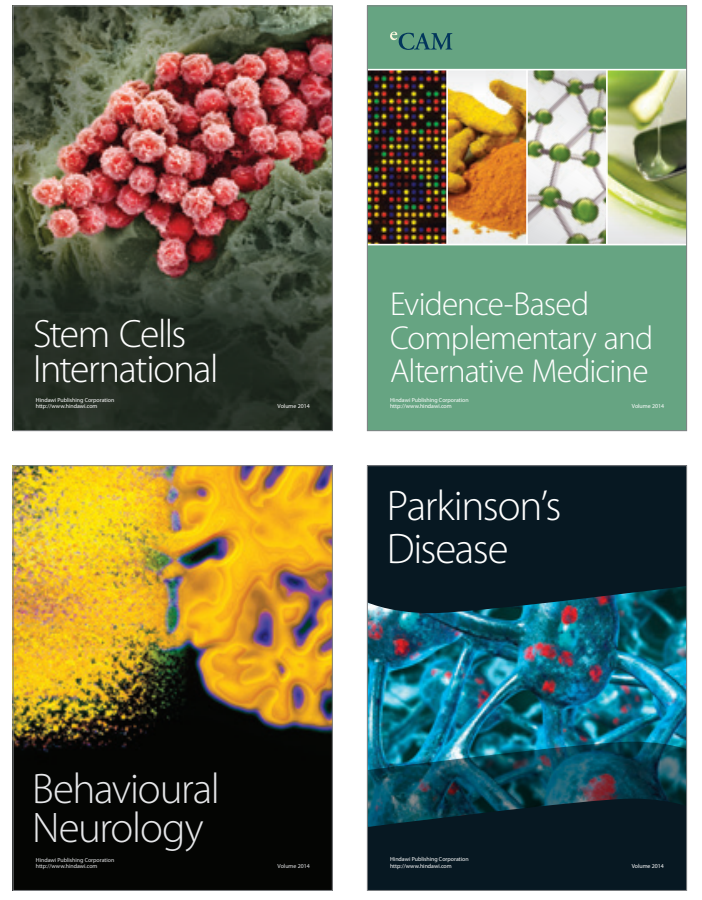
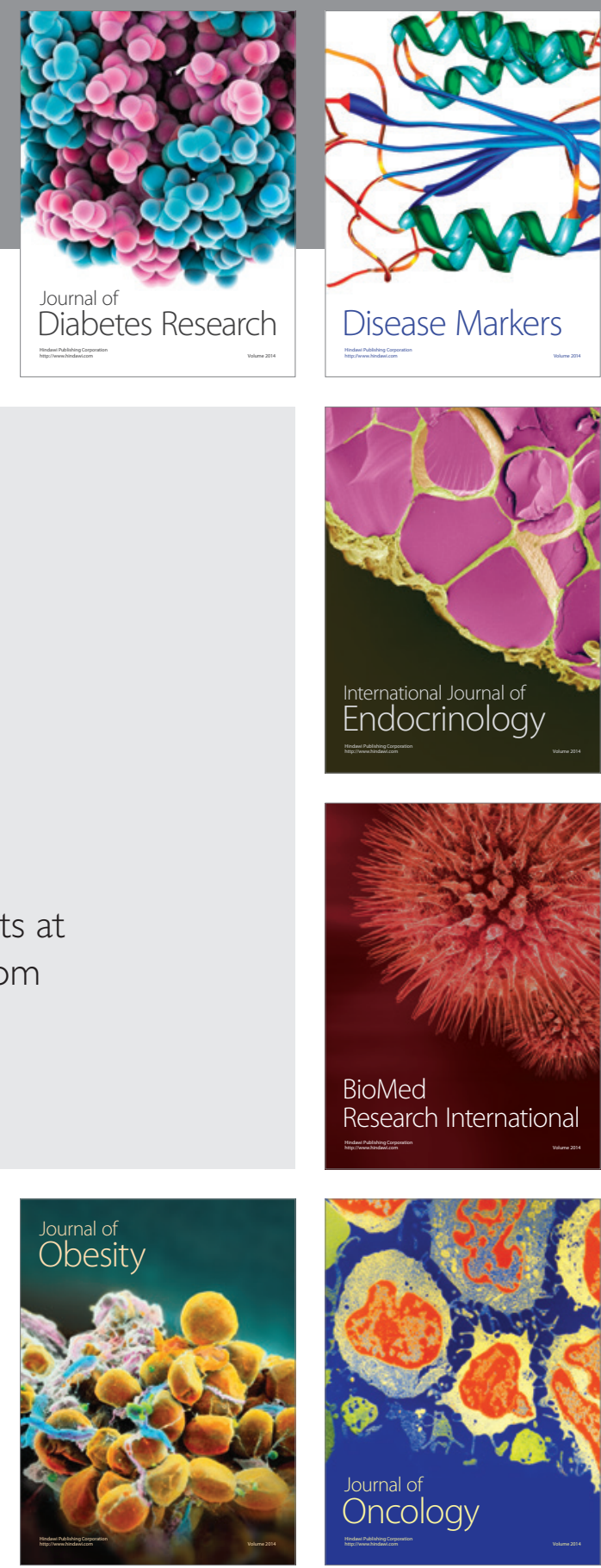

Disease Markers
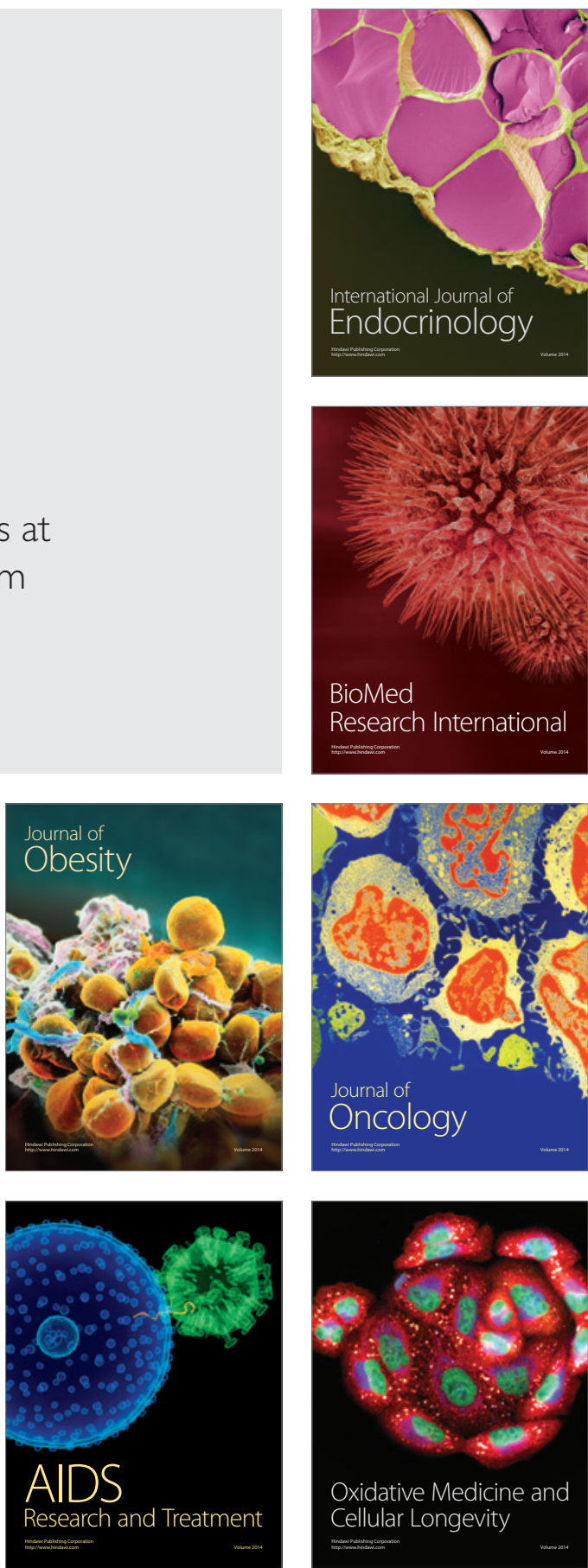